\title{
The role of the Immunisation Adverse Events Clinic at The Children's Hospital at Westmead
}

\author{
Nicholas J. Wood \\ National Centre for Immunisation Research and Surveillance, \\ The Children's Hospital at Westmead \\ Email:Nicholw3@chw.edu.au
}

\begin{abstract}
Specialist immunisation clinics review and manage children who have experienced an adverse event following immunisation and provide advice to parents and health care providers regarding the revaccination of these children. Information collected by these clinics supplement passive surveillance data and allow the investigation of suspected safety signals associated with the delivery of immunisation programs. This paper reviews the role and experience of the Immunisation Adverse Events Clinic at The Children's Hospital at Westmead and identifies areas for development.
\end{abstract}

A clinical service to evaluate and manage children who have experienced an adverse event thought to be associated with immunisation was first established in New South Wales (NSW) at The Children's Hospital at Westmead in 1996. ${ }^{1}$ Similar clinics exist elsewhere in Australia and the United Kingdom, while the United States network of Clinical Immunization Safety Assessment Centers was established in 2001. ${ }^{2,3}$ These clinics assume an important role given community and health care provider concern about adverse events following immunisation. They also collect detailed post-marketing (post-marketing the vaccine has been approved by regulatory authorities for marketing) clinical data on adverse events following immunisation and advise parents and health care providers on revaccination. ${ }^{4,5}$

\section{Clinic aim and function}

The purpose of the Immunisation Adverse Events Clinic at the Children's Hospital at Westmead (the Clinic) is to clinically review children who have had suspected adverse events following immunisation when telephone advice is insufficient and/or where continuation of the routine immunisation schedule is in question. Following review and if indicated - children are vaccinated in the Clinic under supervision. Where there is a risk of a serious adverse event or where there is significant parental anxiety, vaccination is given in hospital as either a day or overnight admission. Some of these parents would have been unwilling to continue the immunisation schedule of their child without the option of hospital observation. Children vaccinated at the Clinic are routinely followed-up by telephone after 72 hours. All adverse events confirmed during consultations are reported to the NSW Department of Health.

\section{Clinic experience}

A total of 809 children were reviewed at the Clinic from January 1997 to December 2009 (Figure 1). Referrals were predominantly from metropolitan Sydney, with only 5\% from rural areas. Of these, approximately three-quarters $(n=649)$ were referred because of a suspected adverse event. The remainder $(n=158)$ were mainly seen for advice concerning future vaccination (e.g. measles-mumpsrubella vaccination in the presence of egg allergy) or due to a chronic medical illness. In some cases, children referred for suspected adverse events were assigned an alternate diagnosis after review in the Clinic. The median age of attendees was 1 year, with one-third aged less than 6 months; half (48\%) were girls.

The Clinic has seen a reduction in adverse events more commonly associated with whole cell pertussis vaccine (hypotonic-hyporesponsive episodes $(n=85)$, seizures $(n=41)$ and large injection site reactions $(n=131))$ following the introduction of acellular pertussis vaccines (Figure 2). Similar to other Australian clinics, revaccination with acellular pertussis vaccine has been successfully completed in children who had experienced severe adverse events such as hypotonic-hyporesponsive episodes, seizures and apnoea. ${ }^{6,7}$ One case of Bell's palsy was seen following human papillomavirus (HPV) vaccination in an adolescent, while most HPV adverse events $(n=18)$ involved non-specific generalised symptoms such as headache, joint pain and syncope.

The Clinic has investigated suspected safety signals such as anaphylaxis and severe allergy following HPV vaccination of adolescents (2007) and fever/febrile convulsions associated with the seasonal influenza vaccine $(2010) .{ }^{8}$ In the case of the increased rate of allergic reactions following HPV vaccination, adolescents were referred for skin-prick testing using a panel of routine allergens to further investigate their reaction and guide future vaccination. ${ }^{8}$ The Clinic contacted parents of children vaccinated with the 


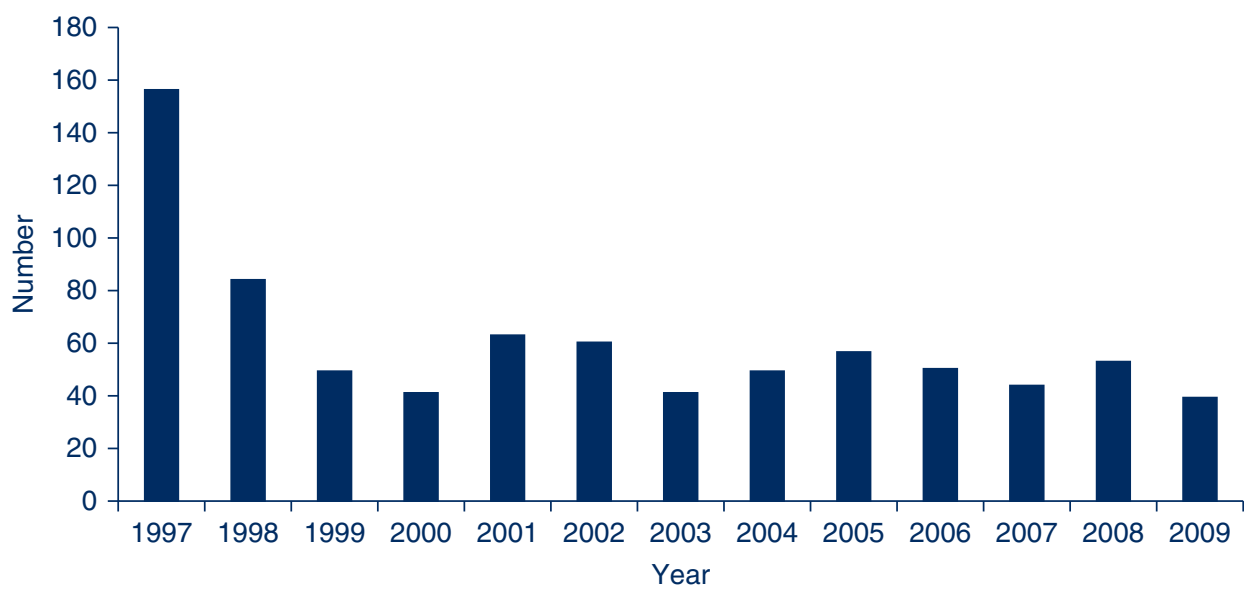

Figure 1. Number of children attending the Immunisation Adverse Events Clinic at The Children's Hospital at Westmead each year for the period 1997-2009.

Source: The Children's Hospital at Westmead Immunisation Adverse Events Clinic database.

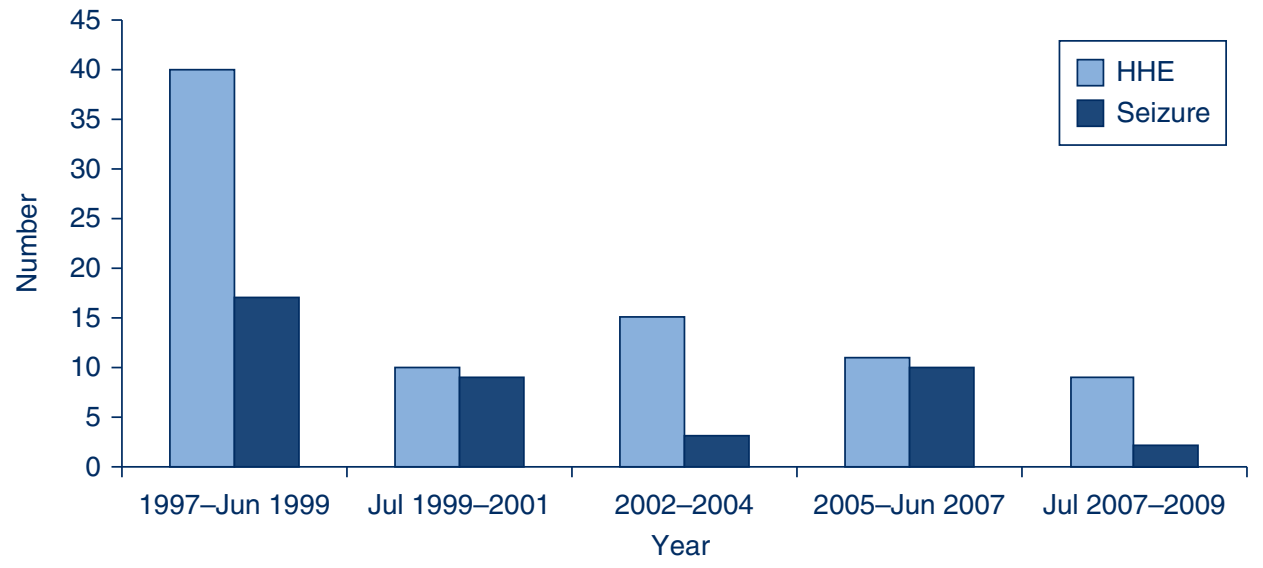

Figure 2. Number of children experiencing vaccine-related hypotonic-hyporesponsive episodes (HHE) or seizure attending the Immunisation Adverse Events Clinic at The Children's Hospital at Westmead each year for the period 1997-2009.

Source: The Children's Hospital at Westmead Immunisation Adverse Events Clinic database.

2010 seasonal trivalent flu vaccine to measure reported fever, febrile convulsions and medically attended illness; this additional data has supported national investigations.

\section{Future development}

Clinical adverse events services for adults and ready access for clinicians and patients outside the Sydney metropolitan area are limited. At present, advice to health care providers concerned about adverse events in adults or for people from regional areas is available by telephone through the Clinic. One group for whom this service is particularly useful is health care workers, given the recent mandatory vaccination program in NSW, as well as parents, for whom there are specific targeted campaigns such as pertussis booster vaccines. A network of relevant medical sub-specialists for clinical review and management of suspected adverse events in older adolescents and adults is under development and will include adult infectious diseases physicians, neurologists, geriatricians and rheumatologists.
Clinical protocols for the management of serious adverse events following immunisation need to be developed. Other plans include: research to examine risk factors for adverse events; long-term follow-up of children who have experienced adverse events; and the training of staff involved in immunisation programs. ${ }^{6,9}$

\section{Conclusion}

The Clinic currently provides an enhanced service at the individual level, promotes parent and provider confidence in continuing the vaccine schedule, reviews those who are in high-risk groups and continues to see parents who are reluctant to pursue vaccination of their children because of fears of adverse events.

For further information contact the Immunisation Clinical Nurse Consultant, Immunisation Adverse Events Clinic, The Children's Hospital at Westmead on (02) 98451433 . 


\section{References}

1. Wood N. Immunisation adverse events clinics. NS W Public Health Bull 2003; 14(1-2): 25-7. doi:10.1071/NB03007

2. Lingam S, Miller CL, Pateman J. Role of an immunisation advisory clinic. Br Med J (Clin Res Ed) 1986; 292(6525): 939-40. doi:10.1136/bmj.292.6525.939

3. Clinical Immunization Safety Assessment network. Centers for Disease Control (CDC). Available from: http://www.cdc.gov/ vaccinesafety/Activities/cisa.html (Cited 30 May 2010.)

4. Ellenberg SS, Chen RT. The complicated task of monitoring vaccine safety. Public Health Rep 1997; 112(1): 10-20.

5. Zhou W, Pool V, Iskander JK, English-Bullard R, Ball R, Wise RP et al. Surveillance for safety after immunization: Vaccine Adverse Event Reporting System (VAERS) - United States, 1991-2001. MMWR Surveill Summ 2003; 52(1): 1-24.
6. Gold M, Goodwin H, Botham S, Burgess M, Nash M, Kempe A Re-vaccination of 421 children with a past history of an adverse vaccine reaction in a special immunisation service. Arch Dis Child 2000; 83(2): 128-31. doi:10.1136/adc.83.2.128

7. Goodwin H, Nash M, Gold M, Heath TC, Burgess MA. Vaccination of children following a previous hypotonichyporesponsive episode. J Paediatr Child Health 1999; 35(6): 549-52. doi:10.1046/j.1440-1754.1999.00419.x

8. Brotherton JM, Gold MS, Kemp AS, McIntyre PB, Burgess MA, Campbell-Lloyd S et al. Anaphylaxis following quadrivalent human papillomavirus vaccination. CMAJ 2008; 179(6): 525-33. doi:10.1503/cmaj.080916

9. Baxter D. The organization, delivery and audit of a specialist immunization clinic. J Manag Med 1995; 9(1): 58-65. doi:10.1108/02689239510080494 